Ramírez, A. y Yáñez, C. (2016). Cuerpos como reexistencia: apertura a nuevas formas de sentido. Mujeres en condición de desplazamiento en Manizales, Caldas. Revista de Sociología y Antropología: VIRAJES, 18 (1), 131-147. DOI: 10.17151/rasv.2016.18.1.7

\title{
CUERPOS COMO REEXISTENCIA: APERTURA A NUEVAS FORMAS DE SENTIDO. MUJERES EN CONDICIÓN DE DESPLAZAMIENTO EN MANIZALES CALDAS*
}

\author{
ALEJANDRA RAMÍREZ ROBLEDO* \\ CARLOS YÁÑEZ CANAL**
}

Recibido: 11 de febrero de 2016

Aprobado: 1 de marzo de 2016

Artículo de Reflexión

\footnotetext{
* El presente artículo se construye tomando como referencia la experiencia investigativa del proyecto "El cuerpo como territorio: apuntes sobre los lugares de reexistencia de mujeres en condición de desplazamiento. Horizontes de sentido desde la gestión cultural"; desarrollado en el marco del programa internacional "Mujeres, diversidad y relaciones sociales de género: desafíos para la construcción democrática de los territorios" del Grupo de Investigación en Identidad y Cultura de la Universidad Nacional de Colombia, y en el marco de la construcción de la línea "Cuerpo, lenguaje y cotidianidad" del semillero sobre antropologías de la violencia, adscrito al Grupo de Investigación Comunicación, Cultura y Sociedad de la Universidad de Caldas.

** Profesional en Gestión Cultural y Comunicativa de la Universidad Nacional de Colombia Sede Manizales. Investigadora del Grupo de Trabajo Académico en Identidad y Cultura de la Universidad Nacional de Colombia, Sede Manizales. Investigadora del semillero sobre antropologías de la violencia, adscrito al grupo de Investigación Comunicación, Cultura y Sociedad de la Universidad de Caldas. E-mail: aleramirezrob@unal.edu.co @ O ORCID: 0000-0002-3974-6155

*** Profesor Asociado de la Universidad Nacional de Colombia, adscrito al Departamento de Ciencias Humanas de la Facultad de Administración de la sede Manizales. Director del Grupo de Investigación en Identidad y Cultura de la Universidad Nacional de Colombia, sede Manizales. E-mail: cyanezc@unal. edu.co (ㅇ ORCID: 0000-0002-4216-3794
} 


\title{
Resumen
}

Este artículo es resultado de varios procesos investigativos que tienen como centro el trabajo desde el cuerpo como territorio con mujeres en condición de desplazamiento. Se inicia con una breve genealogía que establece los referentes de las investigaciones; la segunda parte, focaliza la metodología aplicada, así como las reflexiones en torno a la experiencia de campo. En la tercera parte, se desarrolla la articulación teórica entorno al cuerpo como territorio y la lectura particular con el desplazamiento forzado de mujeres que residen en Manizales, Caldas. Por último, y a manera de conclusión, se pone en tensión el proceso investigativo con las discusiones metodológicas resultantes. El propósito del texto es centrar la atención en la relación de varios procesos investigativos, que han articulado una mirada metodológica particular alrededor del cuerpo en relación con el desplazamiento forzado de mujeres que residen en Manizales, Caldas.

Palabras clave: cuerpo, territorio, reexistencia, mujeres, desplazamiento.

\section{BODY AS REEXISTENCE: OPENNESS TO NEW FORMS OF SENSE WOMEN LIVING IN CONDITIONS OF DISPLACEMENT IN MANIZALES, CALDAS}

\begin{abstract}
This article is the result of several research processes that have as center the work from the body as territory, with women in displacement conditions. It starts with a quick genealogy that stablishes the referents of the researches; the second part, focuses the applied methodology, as well as the reflections around the field experience. In the third part, the theoretical articulation around the body as territory is developed and there is a particular reading about the forced displacement of women that reside in Manizales, Caldas. Finally, and as a way to conclude, tension is placed on the research process with the resulting methodological discussions. The purpose of the text is to center the attention in the linking of various research processes, that have articulated a particular methodological gaze around the body in relation to the forced displacement of women that reside in Manizales, Caldas.
\end{abstract}

Key words: body, territory, reexistence, women, displacement. 


\section{A modo de introducción}

T os referentes fundamentales que han permitido darle continuidad y sentido a los procesos investigativos sobre las mujeres en condición de desplazamiento tienen que ver, por un lado, con el cuerpo en su determinación por diversos sistemas simbólicos y sus múltiples significados; de otro lado, el cuerpo también se asumió como control, regulación, disciplinamiento y normalización; y finalmente, tal vez la más importante en las investigaciones, el cuerpo visto como corporalidad, en la que la creatividad, y la resistencia reexistencia, desempeñan un importante papel transformativo, en términos individuales y colectivos. A partir de este último abordaje, el cuerpo, en el reconocimiento de las vivencias y experiencias, es visto desde lo metodológico como trayectoria de reexistencia, en oposición a la mirada que lo reduce a objeto de estudio.

Como posibilidad investigativa, se generaron espacios de encuentro y desencuentro, de conversación en múltiples dimensiones, las cuales fueron puestas en escena a través de círculos de mujeres. En dicho proceso, se buscaba construir una relación horizontal que permitiera un diálogo entre los investigadores y las mujeres en condición de desplazamiento. En la recreación de historias, como prácticas que enlazan y vinculan, se ha intentado impulsar formas de acción en el reconocimiento sensible, mental y afectivo, así como en la capacidad de movilización de las mujeres asumiendo el cuerpo como reexistencia, buscando la consolidación de una estética-política vinculada con la vida.

En ese sentido, se implementaron los círculos con mujeres en condición de desplazamiento forzado que residen en Manizales en 2015; desde las experiencias que le dieron forma a los procesos investigativos, percibimos que la mayoría de las mujeres que hicieron parte de este proceso fueron desplazadas entre 2000 y 2005 del Magdalena medio, con una mayor presencia del valle del Magdalena, oriente de Caldas, zonas rurales y urbanas de Samaná y Marulanda; además, de la parte alta del occidente de Caldas, zona rural de Riosucio.

Asimismo, desde los lugares de origen se manifestaron claras condiciones de desventaja expresadas en términos de violencia y discriminación de género, exclusión económica, política, social y cultural, las cuales se ven acentuadas por el conflicto armado y el desplazamiento forzado, dando como resultado una mayor pobreza, insatisfacción de necesidades básicas e imposibilidad del disfrute de sus derechos.

Por otro lado, se identificó al desplazamiento forzado en relación con otros hechos violentos, es decir, que estas mujeres pasan por el desplazamiento forzado antecedidas por experiencias de violencia sexual, 
desaparición forzada de familiares, amenaza y persecución. Y, en el proceso de recepción, se continúa con hechos violentos tales como la amenaza, la limpieza social, la estigmatización y la discriminación, así como el desinterés estatal y la imposibilidad del acceso a procesos de atención integrales.

La construcción metodológica de la investigación se asume desde una apuesta estético-política, a partir de una estrategia investigativa de la conformación del círculo de mujeres. Dicho círculo consiste en una práctica colectiva que está relacionada con diversas tradiciones ancestrales de grupos indígenas; ha sido concebida como un proceso de reactualización de la memoria y las narrativas con contenidos sagrados a partir del diálogo y el lenguaje; históricamente, están relacionados con la tradición oral y con la mirada hacia la tierra, desde los elementos fuente de la vida.

\section{Genealogía investigativa}

En el proyecto de investigación "El cuerpo como territorio: apuntes sobrelos lugares de re-existencia de mujeres en condición de desplazamiento. Horizontes de sentido desde la gestión cultural", los siguientes lugares se pueden nombrar como puntos de desenlace donde se construye un entretejido particular de lo teórico y lo metodológico. El primero de ellos tiene que ver con la construcción de la línea "Cuerpos y subjetividades del sufrimiento" del programa internacional de investigación "Cuerpos y subjetividades", que inició en el primer semestre del 2012, con una alianza entre el Grupo de Investigación en Identidad y Cultura de la Universidad Nacional de Colombia, sede Manizales, y el grupo de Sociología del Cuerpo de la Universidad de Michigan; esta línea tenía como objetivo evidenciar las transformaciones sociales del cuerpo, desde la historia del cuerpo de la mujer, aterrizando en la comprensión de las experiencias de sufrimiento en la guerra interna de Colombia.

El segundo, es el programa internacional de investigación “Mujeres, diversidad y relaciones sociales de género: desafíos para la construcción democrática de los territorios, 2015", conformado por universidades de Canadá, Argentina y Colombia, y cuyo objetivo general era el reconocimiento y el crecimiento de la capacidad de acción y de movilización de las mujeres en el desarrollo democrático, a través del trabajo conjunto entre los investigadores y comunidades de los distintos países. En Colombia, desde el Grupo de Investigación en Identidad y Cultura se desarrolló el proyecto "Cuerpo, mujeres y desplazamiento forzado", y en este se realizó la "V Semana de la Alteridad: El cuerpo como territorio: las mujeres y el desplazamiento forzado en Colombia". En este encuentro internacional, 
grosso modo, se intentó desarrollar una mirada desdelas formas corpóreas que aparecían en las mujeres que pasaban por la experiencia del desplazamiento forzado en Colombia.

El tercero hace referencia al "semillero en antropologías de la violencia", adscrito al Grupo de Investigación "Comunicación, Cultura y Sociedad" de la Universidad de Caldas. En este proceso se ha desarrollado una línea de investigación sobre cuerpo, lenguaje y cotidianidad en la cual se intenta puntear un camino desde la inflexión de la mirada, hacia la construcción de un trabajo en las antropologías de la violencia, con los puentes éticos, teóricos, estéticos y metodológicos, que, al entender particularmente los niveles de complejidad existentes en esta relación, permitan interconectar el cuerpo, el lenguaje y la cotidianidad como una apuesta de interpelación a las múltiples realidades que se abordan desde esta línea.

\section{Convirtiendo la experiencia en texto. Resonancias}

Nota sonora: Hemos sentido miedo del despojo, miedo al desarraigo, temor al desamparo como condición impuesta de la existencia, nos han quebrado la mirada con el horror, nos han dejado los lugares en la continuación de la muerte, han hecho de nuestro cuerpo el lienzo de lo monstruoso y aun así, aquí estamos, seguimos siendo la huella de la vida y el rostro en el rastro de lo perverso...

En esta parte, vamos a intentar articular nuestras voces con la de las mujeres en condición de desplazamiento, en la idea de hacer un diálogo en este texto, como una forma de traducir nuestra experiencia pensando en la idea de que "el sentido más profundo del yo, se experimenta cuando uno es con el otro" (Braidotti, 2004, p. 46). En tal sentido, comenzaremos con situar los procesos metodológicos de este trabajo, pues aquí encontramos una fuerza potente para nuestra experiencia, y de manera seguida pondremos en conversación las reflexiones entorno a lo vivido en trabajo de campo.

En el planteamiento metodológico que se puso en escena en el trabajo de campo, surgen lo que diferentes investigadores, entre ellos Juan Pablo Aranguren (2008), Sandro Jiménez (2008) y Alejandro Castillejo (2000), han denominado como "los dilemas éticos de la investigación sobre violencia", al insistir que en temas relacionados con experiencias o situaciones límites como la del desplazamiento forzado, el investigador debe partir de una sensibilidad diferente, de reinventar sus propias metodologías de investigación y de ponerse en el lugar del otro, pues cuando nos encontramos ante experiencias inacabadas, de fractura y discontinuidad, van a aparecer diferentes formas de olvido y silencio que el investigador debe estar equipado para reelaborar y traducir. 
Nota sonora: es sentirse bien, venir y compartir un rato de los que uno sabe, uno se siente importante... a mí me gusta venir y verlas.

La propuesta metodológica construida para el proyecto de investigación, consiste en un trabajo experiencial, que permita comprender la experiencia como propuesta para identificar las reexistencias como una posibilidad, como un horizonte, que inscribe el cuerpo como un territorio: el espacio, la subjetividad y la identidad, entonces, se convierten en los procesos en los que nos encontraremos para darle forma a esta intuición en los círculos de mujeres.

Nota sonora: a uno lo miran todo feo como si fuera una enfermedad que contagia... Nota sonora: yo fui desplazada hace muchos años, pero todavía cuando le digo a alguien me hace a un lado...

Las estrategias investigativas que se aplicaron se caracterizan por tener la mirada puesta en la experiencia y en la voz de las mujeres. La forma en la que trabajamos fue a partir de dichos círculos de mujeres, metodología que pretende a través de la experiencia reflejada en sus propias palabras, en sus silencios y en los saberes de todas las participantes, crear otro tipo de encuentro, un espacio de conversación, que permita que cada una pueda mirarse desde adentro, reconocerse en las otras y asumir nuevos retos en la propia construcción de mundo. Así, este proceso metodológico resulta "desestructurado"1, el investigador aparece como un mediador, como un sujeto político, activo, que se vincula en un escenario en donde la guía, la hoja de ruta, pasa por el diálogo y la interlocución entre las participantes. Esta es una apuesta por los procesos colaborativos, y por la generación de redes sociales que ayuden a recobrar la confianza y el sentido colectivo entre las mujeres que, a pesar de habitar la ciudad de Manizales desde hace tantos años, aún representan la periferia de la periferia como la negación de un lugar social (Castillejo, 2000)2.

\footnotetext{
${ }^{1}$ Desde una mirada científica y tradicional de los estudios sociales; sin embargo esto no es una investigación desde las ciencias sociales, sino desde las humanidades, con un reconocimiento al trabajo interdisciplinar y al empírico.

${ }^{2}$ Alejandro Castillejo en su libro Poética de lo otro, hace un recorrido por lo que podríamos pensar como el proceso inacabado del desplazamiento forzado, entendiendo que los puntos de fuga de esta experiencia pasan por las indefiniciones; así pues, uno de esos puntos de fuga se refiere a la recepción, que se convierte en otra experiencia de violencia con la estigmatización, la marginación y las formas de vulnerabilidad a las que se ve expuesto el desplazado o la desplazada. A esto es a lo que se refiere cuando habla del desplazado como un ser sin un lugar social, al que se le delimita la existencia aun sin estar muerto.
} 
Nota sonora: pues vean... a mí me sacaron de mi casita, pero no me quitaron las ganas de salir adelante, sigo viva y por duro que sea pues tengo manos pa' trabajar... pa' darle de comer a los hijos y reprenderlos pa' que no se le vayan a torcer a uno...

Los círculos de mujeres son un reflejo que encuentra su raíz en los procesos de consolidación de acuerdos, puntos de vista y desahogo de las mujeres de comunidades afro y de comunidades indígenas que establecían, desde su cotidianidad, momentos en los que la división del trabajo encontraba labores que se asignaban solo a mujeres; el lavado de prendas y herramientas en río o la preparación de alimentos son un ejemplo en general de labores que permitían estos encuentros.

Desde teóricas decoloniales (Braidotti, 2010) que han surgido con influencia en América latina, existe una corriente que tiene que ver con la renovación de lo femenino como quehacer político en el feminismo del sur; de allí, viene dándose un retorno a los círculos de mujeres con la construcción de redes, principalmente desde los trabajos colaborativos entre organizaciones comunitarias de mujeres y trabajos barriales en diferentes zonas.

Argentina y México son países que han abanderado la implementación de círculos de mujeres en las áreas comunitarias y organizativas, así como en un proceso de consolidación de estos encuentros, llevándolos a la práctica académica como escenario metodológico dentro de la investigación cultural.

Por su lado, en Colombia se retorna a los círculos de mujeres de manera incipiente desde hace 5 años por las organizaciones, colectivos feministas y procesos de empoderamiento que están relacionados con los cantos al agua y a la Pachamama. Así, la incidencia dentro de la práctica académica en el país no está consolidada ni considerada por los y las investigadores en general; sin embargo, existen algunos esfuerzos que comienzan a ser visibles dentro de la investigación, como la Universidad Autónoma de Manizales y la Universidad Nacional de Colombia.

Los círculos de mujeres consisten en ritualizar un encuentro a través de elementos como los alimentos, el fuego y la palabra. Se trata de construir un grupo en el que el principio de colaboración, y a través del compartir, se traduce desde la forma en la que cada una da a la otra una porción de sí. En dicho proceso, los alimentos juegan un papel importante, ya que son una muestra de su raíz y del lugar del que fueron desplazadas. Las mujeres siguen cocinando sus alimentos tradicionales, es decir, que sus gastronomías no se desplazan de sus manos y, en ese sentido, compartir los alimentos se hace una muestra de resistencia y de re-existencia de sus saberes.

Al fuego se le da el sentido de la escucha y de la construcción de diálogo. El ambiente se encuentra intervenido por velas y en el círculo 
cada una de ellas tiene su propia vela, que es la forma en la que anuncia su participación con la palabra; cuando una de ellas termina de hablar, o de hacer su intervención, le da fuego a la siguiente mujer que va a participar. Cada tema que es tratado en el círculo de mujeres, es dirigido por la espontaneidad de su sentir. Los diálogos tienen la idea de darnos la posibilidad de recorrer las experiencias en común o discontinuas sin la centralidad en el hecho violento y en sus causas, sino, más bien, en la forma en la que sus vidas cotidianas se han transformado. En esa medida, los lugares por los que pasa su reconfiguración de la cotidianidad y, para este caso, la forma en la que esto permite que el cuerpo se vuelva su territorio de reexistencia.

Nota sonora: Demostrar una violación en este país, es volver a ser violada...

Por otro lado, y refiriéndose ya con lo que tiene que ver en la experiencia de trabajo de campo, el círculo que se construyó desde este proceso, permitió que, en los diálogos de los distintos encuentros, los temas se diversificaran; cada una de las mujeres contó su propia historia, la forma en la que recuerda y actualiza su pasado, las ausencias y las presencias que conforman el hoy. Pasamos por las gastronomías, por las formas de educar las hijas y los hijos, por la forma en la que se decora el hogar y por la forma en la que se sigue viviendo... después de la experiencia de violencia... después del desplazamiento forzado... después de la violación sexual... después de vivir la desaparición forzada del compañero... de un hijo...

Nota sonora: aquí vinieron una vez y me preguntaron un montón de cosas, me dijeron que me iban a ayudar para que me metieran a ese registro y nada... nunca se volvieron a asomar...

De esta forma hemos logrado construir un itinerario diferente, donde el testimonio dado por la "mujer desplazada" no se comporta como citas ubicadas estratégicamente en el texto para darle credibilidad y soporte a lo que el investigador muestra como resultado de su investigación; su historia no es parte de la verificación y del respaldo de la traducción del investigador. Por el contrario, en este proceso los diálogos, construidos en los círculos con mujeres en condición de desplazamiento, han permitido una serie de rutas, caminos e intuiciones que han sido el pincel, el acto creativo para la transformación de este trabajo en un proceso investigativo. En este artículo, más que fragmentos de los testimonios, aparecen notas sonoras que son la extensión de silencios y de nuestra permanencia... 
Nota sonora: yo ya no soy víctima, no soy una víctima, ni siquiera me ayudaron cuando lo era...

El acompañamiento y el hacer parte de los círculos, permitieron ver la forma en que las mujeres en condición de desplazamiento, viven en una frontera constante, los lugares de recepción no son sus hogares, y el que era su hogar ya no lo es; sin embargo, en la lucha de supervivencia, en el flujo del devenir, sus saberes y pertenencias simbólicas pasan por intervenciones rizomáticas, es decir, además de multiplicarse, se ramifican y amplían los modos de relación e interacción. Allí es donde aparecen las formas de reexistencia: las mujeres, en su intento por reconstruir su vida, comienzan un proceso de "adaptación" y se perfilan en interconexiones con el espacio y el contexto que se ven atravesados por conflictos que resultan de procesos de desarticulación y rearticulación de los significados simbólicos.Así, en el enfrentamiento con el lugar de recepción, las mujeres logran apropiarse de una estética de la vida que tiene que ver, y que pasa fundamentalmente por la resistencia.

Para las mujeres que construyeron este círculo, las referencias que las vinculan con sus tradiciones culturales y sus historias individuales y colectivas, pasan por experiencias que rompen con la memoria social incorporada. La trama social (la familia, la clase, el género, la etnia), en que se han consolidado su mundo de sentidos y significados, se disuelve y pasa a hacer parte del mundo de los recuerdos y las remembranzas, debido a la imposibilidad del retorno, ya sea porque los lugares de los que fueron desplazadas aún siguen siendo acontecidos por la violencia o porque el haber sido desplazadas desde 2000 ó 2005, no se sometieron al proceso médico, legal o jurídico, que se requería para ser reconocidas como víctimas, o porque después de 10 años o más de sucedido el desplazamiento, han logrado establecerse y reconocerse desde otros roles que no son los que tenían asignados en su lugar de origen, esto es en el campo el rol de cocineras y cuidadoras de hogares, y en la zonas urbanas, amas de casa.

En este sentido, esta investigación se preocupó por el después del desplazamiento, así como otras investigaciones se han preocupado par la narrativa de la víctima. Hemos decidido construir desde las sobrevivientes, dándole todo el sentido a su historia de vida, a la huella del desplazamiento, pero mirando la forma en la que el hoy nos exige detenernos, tocar un pedacito del mañana, de ese que es tan impredecible, para darnos cuenta que contar, que narrar como forma de reinvención, pasa también por la forma en la que se reconstruye la cotidianidad, pasa por las reexistencias, desde las formas de organización y de lucha, como desde las subjetividades de las personas en condición de desplazamiento. 


\section{Articulación teórica entorno al cuerpo como territorio y la lectura particular con el desplazamiento forzado de mujeres que residen en Manizales}

Dejando enunciados los lugares que construyen la genealogía de este artículo y el proceso metodológico aplicado, es necesario dar cuenta del marco teórico sobre el cual ha sido posible pensar la forma en la que la experiencia del desplazamiento forzado en mujeres, que ahora residen en Manizales, logra configurar el cuerpo como un territorio de re-existencia, en la medida en la que se reelabora el pasado y su vínculo con éste, a partir de las prácticas cotidianas.

La condición humana es corporal. Materia de identidad en el plano individual y colectivo, el cuerpo es espacio que ofrece vista y lectura, permitiendo la apreciación de los otros. Por él somos nombrados, reconocidos, identificados a una condición social, a un sexo, a una edad, a una historia.... (Le Breton, 2005. p. 17)

El cuerpo es un lenguaje universal con diversificaciones propias de lo sensible en el mundo de lo cotidiano; el cuerpo, de acuerdo con Nancy, es:

la mediación sensible en la que un sentido se hace sentido, esto es, se siente. En esta presentación sensible, el sentido se expone en su salida de sí mismo, en su vaciamiento o en el auto-espaciamiento, dado que el límite sensorial en el que lo sentimos nunca coincide con el sentido como tal, con su interioridad o su sí mismo (soi). Es importante anotar que en este movimiento de partida de sí del sentido, partida que es auto-separación y sustracción, el cuerpo es tanto el escenario de su exposición como la interioridad que allí se expone (Nancy, 1993). Esto quiere decir que lo que se expone no se distingue del medio de su exposición, no lo antecede: el cuerpo es en y como su propio espaciamiento o su retirada ya que aquello que en él se expone (su sentido) sólo puede serlo en y como la exposición misma. (Pérez Moreno, 2014. P. 1-2)

Así, el trascender la idea de los sentidos, como hacedores de lenguaje, nos permite mirar el cuerpo desde un lugar distinto; por ejemplo: el tocar, en apertura y extensión a la mirada de los sentidos. Jacques Derrida en el libro El tocar, Jean-Luc Nancy habla de le toucher como: "el tacto y tocado en el tocar" (Derrida, 2011, p. 20). Aquí se hace evidente el sentido transdimensional del cuerpo bajo tres afirmaciones "1) el órgano del tacto es interno. 2) Que la carne no es más que un intermediario del tocar 3) 
que el tocar tiene por objeto lo tangible y lo no-tangible" (Derrida, 2011, p. 24); con esta última afirmación llegamos desde Derrida a una pregunta que permite un encuentro íntimo de la subjetividad con la multiplicidad del lenguaje vivo, eso es un lenguaje también hecho carne: ¿cómo tocar en lo intocable? (Derrida, 2011, p. 25).

El cuerpo es el lugar de convergencia de las experiencias que construyen las realidades, es el agente y el agenciamiento, donde nacen y se transforman, donde se dinamizan y donde interactúan, prácticas y elaboración de sentidos, así como de las significaciones y procesos simbólicos que le dan lugar a lo cotidiano. "En la vida cotidiana nos encontramos sumidos en la experiencia sensible del mundo. Todos los sentidos se encuentran permanentemente en actividad, intercambiando conjuntamente sus informaciones en la conducción de la existencia" (Le Breton, 2005, p. 62).

Esto último, puede entonces remitirnos a las formas que configuran la cotidianidad como transformadora del cuerpo en el campo de lo político. Para Michel Bernard, parafraseando a Paul Valéry, en el cuerpo un fenómeno ambivalente dice: "si nuestro cuerpo es el "órgano de lo posible" lleva también y simultáneamente el sello de lo inevitable... De manera que toda reflexión sobre el cuerpo, es quiérase o no, ética y metafísica: proclama un valor, indica una cierta conducta y determina la realidad de nuestra condición humana" (Bernard, 1985, p. 24).

Esta investigación centró su mirada en el cuerpo como corporalidad, que será la forma en la que veremos un entramado con el cuerpo y la experiencia, teniendo en cuenta que el cuerpo como territorio es el lugar en donde pasa la experiencia, donde se inscribe y se construye un itinerario desde la historicidad y se compone la antesala del mañana; el cuerpo es el lugar que el desplazado no dejó y del que no ha sido sacado, es el lienzo por el que pincela la huella el desplazamiento y por dónde camina la mirada del hoy.

De esta forma, el marco de análisis a partir del tocar, le toucher, implica, como lo propone el profesor Alejandro Castillejo (Castillejo, 2010) ${ }^{3}$, una inflexión en la mirada, replanteando el lenguaje que se articula para traducir la experiencia de violencia del otro y las categorías de análisis que el investigador construye. Así, el interés específico en el cuerpo como territorio y en el desplazamiento desde las subjetividades, identidades y espacios desde la perspectiva que posibilita la biocultura ${ }^{4}$, viendo como un

\footnotetext{
3 "Guerra, cotidianidad y órdenes globales" (Alejandro Castillejo, 2010). "Poética de lo otro. Antropología de la guerra, la soledad y el exilio interno en Colombia" (Alejandro Castillejo, 2000).

${ }^{4}$ Concepto desarrollado por la profesora Rubiela Arboleda en su libro "El cuerpo: huellas del desplazamiento" (2009).
} 
referente el trabajo sobre desplazamiento de Rubiela Arboleda (Arboleda, 2009), se propone además centrar la mirada en el mundo de los encuentros cara a cara, es decir, un retorno a la vida cotidiana para comprender el desplazamiento como experiencia y categoría, como aparece en la propuesta teórica y metodológica que articula el profesor José Castro ${ }^{5}$ (Castro, 2012) en su texto "El desplazamiento forzado en Colombia: experiencia y categoría. Notas al margen para una antropología de lo efímero." 6

La experiencia del desplazamiento forzado, desde la perspectiva de la biocultura, inscribe una huella particular en el cuerpo ${ }^{7}$, un memorando específico en la subjetivación, que hace de ella un proceso inacabado donde el sujeto se reinventa y se define constantemente al estar inmerso

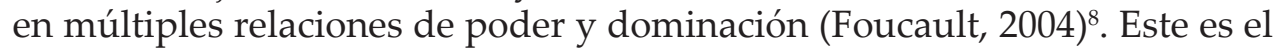
lugar en donde cobra sentido enunciar como re-existencia los procesos que se refieren a las formas en las que se reconfigura la vida cotidiana ${ }^{9}$, teniendo en cuenta que este es el escenario en el que se construye y recrea la fractura de la violencia en el yo, en el cuerpo.

En general, los autores y trabajos anteriormente mencionados, así como un recorrido por los trabajos de investigación realizados en la región por el profesor Mario López ${ }^{10}$, con una particular atención en la política pública para la atención al desplazamiento en el departamento de Caldas; las profesoras María Cristina Palacio y María Rocío Cifuentes ${ }^{11}$, que han dedicado gran parte de su trabajo investigativo a comprender las dinámicas del conflicto armado en Caldas, y específicamente la población en condición de desplazamiento con algunos marcos de perspectiva de

\footnotetext{
${ }^{5}$ El texto "El mapa como mediación visual y la voz como itinerario: instantáneas del conflicto en Caldas" del profesor José Castro de la Universidad de Caldas, también construye el antecedente de este trabajo. 6 "Desplazamiento forzado, experiencia y categoría: notas al margen para una antropología de lo efímero" (José A. Castro, 2012. Texto inédito).

${ }^{7}$ Desde la perspectiva de la biocultura, es posible pensar en el cuerpo como un lugar de significación que permite pensar la subjetividad, la identidad y el espacio.

${ }^{8}$ Esta idea pensada desde la interpretación de "El sujeto y el poder" (Michael Foucault, 2004).

${ }^{9}$ En este proyecto, el mundo de la vida cotidiana es una expresión que requiere una breve descripción. En este sentido, lo que se denomina aquí vida cotidiana, no hace referencia a lo que pasa todos los días y se vuelve rutinario, normal, y autoevidente al punto de la desaparición. Este es quizás el contenido que coloquialmente, incluso en la misma investigación social, se le asigna a la palabra: lo ordinario, lo que acaece todos los días, la trivialidad e irrelevancia de la vida, lo que no es extraordinario. Vida cotidiana tiene que ver, más bien, con el universo de encuentros cara-a-cara que se gestan entre las personas en muy diversos contextos sociales (Bauman, 1993; Goffman, 1959; Castillejo, 2012).

10 "Las políticas públicas en materia de desplazamiento forzado por la violencia en Colombia"(Mario Hernán López, Revista de ciencias sociales y humanas, 2007). "La política pública para la atención al desplazamiento forzado en el departamento de Caldas y en los municipios de Riosucio y Samaná (19972006)" (Mario Hernán López, Revista Luna Azul, 2009).

11 "Departamento de Caldas: su configuración como territorio de conflicto y desplazamiento forzado" (María Cristina Palacio Valencia, Rocío Cifuentes Patiño, trabajo social, 2005). "El conflicto armado y el desplazamiento forzado en Caldas: crisis de la institucionalidad familiar" (María Cristina Palacio Valencia) Revista Latinoamericana de Ciencias Sociales, Niñez y Juventud, 2005).
} 
género, permitieron una guía para este proyecto de investigación que pasa por la mirada que se articula para traducir el desplazamiento forzado y por los antecedentes que le han dado lugar a esta investigación.

\section{A manera de conclusión}

El cuerpo es piel límite de lo ilimitable, frontera porosa y fluida de nuestro ser al mundo con el mundo, intercambio vital-corporal-emotivocognitivo con otros (Yáñez, 2010). El cuerpo como territorio aparece en movilidad constante, es un territorio nómada en donde aparecen las negociaciones de las dimensiones de lo íntimo y lo privado; esto entendiendo que la experiencia de violencia, la que antecede el desplazamiento forzado,y/o este mismo, modifican la forma en la que la mujer establece estas dimensiones en relación con la corporalidad, en tanto trama de lo social y lo experiencial. En ese sentido, aparece una conclusión de esta apuesta investigativa; el cuerpo como método, permite la construcción de estrategias metodológicas en las que se revitaliza la memoria, no en términos de la memoria del olvido o el olvido de la memoria, sino en la posibilidad de una memoria viva y dinámica, que aparece y atraviesa los cuerpos. Se habla, pues, de una memoria de la corporalidad en donde la afectividad se hace parte de la estética de la vida, para construir solidaridad y redefinir su propia experiencia y la particularidad de su historia en encuentros como los círculos de mujeres.

El proceso de desplazamiento, específicamente el momento de recepción, que posee características violentas y traumáticas para las desplazadas, además de incidir en las condiciones demográficas, sociales, institucionales y culturales de un país, genera una ruptura de los límites, lo que conlleva un "vivir en crisis", planteándose una reflexión epistémica, ética y política, en donde se asuma una potencia en términos de las reacciones de resistencia y re-existencia. Así, la propuesta del "vivir en crisis" se asume como una noción de agencia, la cual permite superar la mirada dualista referente a lo micro y lo macro de las ciencias sociales, para potenciar la manera en la que los sujetos reaccionan al sufrimiento social (Vignolo, 2009). “Esta aproximación permite examinar situaciones liminales diferentes, desde aquellas en las que los sujetos sociales cuentan con recursos afectivos, simbólicos y materiales para la transformación de sus circunstancias, hasta aquellas en que los sujetos son avasallados por las condiciones de opresión" (Vignolo, 2009, p. 46).

Así, el círculo de mujeres aparece como posibilidad de generar una trama corpo-afectiva que se oponga al drama y a la revictimización, 
de manera que se centre en las dinámicas de re-existencia. Estos círculos son encuentros colectivos de mujeres que han pasado por la experiencia del desplazamiento, y buscan establecer procesos de diálogo, espacios de conversación que plantean el reconocimiento de sus experiencias, sus narrativas, sus saberes, sus raíces y destellos del lugar del que fueron desplazadas, los cuales aparecen en su intimidad y en la posibilidad de encontrarse con aquellas otras mujeres que también hacen parte de su historia.

Las luchas por intervenir la regulación, por modificarla en favor de determinadas preferencias, se libran también en una arena en la que concurren fuerzas de carácter subjetivo, es decir, estético. Es, entonces, en función de lo que las personas o grupos de intereses subjetivos específicos entienden por vida como se entablan tales luchas. $\mathrm{Y}$ en luchas por vivir la vida, por gestionarla de formas concretas, el cuerpo se presenta como baluarte. El cuerpo aparece allí como algo más que el mero resultado de las visiones anatómica y fisiológica que disciplinaron en el panóptico y sus tecnologías; resulta ser algo más que la dispersión de riesgos, información y cifras que regulan las actividades cotidianas de las personas, para traducirlas en conceptos estadísticos: el cuerpo se postula como esencia misma de humanidad, entramado corpo-afectivo en que los seres humanos se reconocen sensible, mental y afectivamente. En estas luchas, el cuerpo no se esgrime -a la manera académica- como producto del discurso, sino estéticamente como la expresión misma de la vida." (Pedraza Gómez, 2007, p. 386).

Para terminar, lo que debió ser el comienzo... 
Mano fuerte va barriendo, pone leña en el fogón Mano firme cuando escribe una carta de amor Manos que tejen haciendo nudos Manos que rezan, manos que dan

Manos que piden algún futuro $\mathrm{Pa}^{\prime}$ no morir en soledad jay! ;Ay!... Mano vieja que trabaja Va enlazando algún telar Mano esclava va aprendiendo A bailar su libertad Manos que amasan curtiendo el hambre

Con lo que la tierra les da Manos que abrazan a la esperanza De algún hijo que se va ;ay! ;Ay!... Manos de mujeres

Que han parido la verdad Manos de colores aplaudiendo algún cantar Manos de mujeres Que han parido la verdad Manos de colores aplaudiendo algún cantar Manos de mujeres

Que han parido la verdad Manos de colores aplaudiendo algún cantar ;ay! ;Ay!... Mano fuerte va barriendo, pone leña en el fogón Mano firme cuando escribe una carta de amor

Manos que tiemblan, manos que sudan

Manos de tierra, maíz y sal

Manos que tocan dejando el alma

Manos de sangre, de viento y mar

Manos que tiemblan, manos que sudan

Manos de tierra, maíz y sal

Manos que tocan dejando el alma

Manos de sangre, de viento y mar ;ay! ;Ay!...

\section{Marta Gómez, Cantautora colombiana.}




\section{Referencias bibliográficas}

ACNUR. (2013). Informe Nacional de Desplazamiento Forzado en Colombia, 1985 a 2012. Bogotá.

Arboleda, R. (2009). El cuerpo: huellas del desplazamiento. Medellín: Hombre Nuevo Editores.

Aranguren, J.P. (2008) El investigador ante lo indecible y lo inenarrable (una ética de la escucha), Nómadas No.29, Bogotá, Universidad Central.

Barreiro Martínez, A. (2004). La construcción social del cuerpo en las sociedades contemporáneas. En: Papers, Revista de Sociología, Vol. 73 (2004). Barcelona: Universitat Autònoma de Barcelona.

Bernard, M. (1985). El cuerpo, un fenómeno ambivalente. Barcelona: Paidós.

Braidotti, R. (2004). Feminismo, diferencia sexual y subjetividad nómade. Barcelona: Gedisa.

Butler, J. (2007). El género en disputa. El feminismo y la subversión de la identidad. Barcelona: Paidós.

Castillejo, A. (2012). Guerra, cotidianidad y órdenes globales. Notas antropológicas para una relectura de la violencia en Colombia. Popayán: Universidad del Cauca, Instituto Colombiano de Antropología, ICANH.

Castillejo, A. (2000). Poética de lo otro. Antropología de la guerra, la soledad y el exilio interno en Colombia. Bogotá: Colciencias.

Castro, J. (2012). El desplazamiento forzado en Colombia: experiencia y categoría. Notas al margen para una antropología de lo efímero. Texto inédito.

Citro, S. (2009). Cuerpos significantes. Travesías de una etnografía dialéctica. Buenos Aires: Editorial Biblos.

Derrida, J. (2011). El tocar, Jean Luc-Nancy. Buenos Aires:.Amorrortu Editores.

Duch, Ll., Melich, J. C. (2005). Escenarios de la corporeidad. Antropología de la vida cotidiana. Madrid: Editorial Trotta.

Goffman, E. (2006). Estigma. Buenos Aires:.Amorrortu Ediciones.

Glissant, E. (1990). Poetique de la relation. Paris: Gallimard.

Jiménez, S. (2008). Etnografía de la crisis: sobre algunos debates y una práctica de investigación en contextos de violencia política», Nómadas No.29, Bogotá, Colombia, Universidad Central.

Le Breton, D. (2005). Cuerpo sensible. Santiago de Chile : Metales Pesados.

López, M. H. (2007). Las políticas públicas en materia de desplazamiento forzado por la violencia en Colombia. Colombia: Revista Zona, No. 2 (2007).

López, M. H. (2010). La política pública para la atención al desplazamiento forzado en el departamento de Caldas y en los municipios de Riosucio y Samaná (1997-2006). Manizales: Revista Luna Azul, No. 30 (2010).

Nancy, J. L. (1993). The Birth to Presence. Trad. Brian Holmes. Stanford : Stanford University Press.

Nancy, J. L. (2004). El cuerpo como objeto de un nuevo pensamiento filosófico y político. Barcelona: Anthropos.

Pedraza Gómez, Z. (2007). Políticas y estéticas del cuerpo en América Latina. Bogotá: Uniandes.

Pérez Moreno, J. D. (2014). Filosofía del tacto de Jean-Luc Nancy, Ley y violencia. Bogotá: Universidad de los Andes.

Pérez Moreno, J. D. (2014). Recuperado de: http://grupoleyyviolencia.uniandes.edu.co/ Web/documentos/Proyecto\%20Juan\%20Diego\%20P\%C3\%A9rez.pdf. 20/12/2015

Sabido, O. (2012). El cuerpo como recurso de sentido en la construcción del extraño. Una perspectiva sociológica. Madrid: Ediciones Sequitur. 
Suárez Cardona, L. F. (2013). Mal y sufrimiento humano. Un acercamiento a un problema clásico. Bogotá: Anábasis.

SIPOD, Agencia Presidencial Para La Acción Social, 2012, Bogotá.

Vignolo, P. (2009). Ciudadanías en escena. Bogotá: Universidad Nacional de Colombia.

Yáñez Canal, C. (2010). Viaje al "uno en la multiplicidad". La identidad personal y sus "sí mismos". Bogotá: Universidad Nacional de Colombia.

Yáñez Canal, C. (2014). La identidad personal entre afectos y afectaciones. Manizales: Universidad Nacional de Colombia. 